\title{
In vivo efficacy of artesunate- amodiaquine and artemether-lumefantrine for the treatment of uncomplicated falciparum malaria: an open-randomized, non-inferiority clinical trial in South Kivu, Democratic Republic of Congo
}

Marit de Wit ${ }^{1 *}$, Anna L. Funk ${ }^{1}$, Krystel Moussally', David Aksanti Nkuba', Ruby Siddiqui ${ }^{2}$, Karla Bil $^{1}$, Erwan Piriou' ${ }^{1}$, Aldert Bart ${ }^{3}$, Patrick Bahizi Bizoza ${ }^{4}$ and Teun Bousema ${ }^{5,6}$

\begin{abstract}
Background: Between 2009 and 2012, malaria cases diagnosed in a Médecins sans Frontières programme have increased fivefold in Baraka, South Kivu, Democratic Republic of the Congo (DRC). The cause of this increase is not known. An in vivo drug efficacy trial was conducted to determine whether increased treatment failure rates may have contributed to the apparent increase in malaria diagnoses.

Methods: In an open-randomized non-inferiority trial, the efficacy of artesunate-amodiaquine (ASAQ) was compared to artemether-lumefantrine (AL) for the treatment of uncomplicated falciparum malaria in 288 children aged 6-59 months. Included children had directly supervised treatment and were then followed for 42 days with weekly clinical and parasitological evaluations. The blood samples of children found to have recurring parasitaemia within 42 days were checked by PCR to confirm whether or not this was due to reinfection or recrudescence (i.e. treatment failure).
\end{abstract}

Results: Out of 873 children screened, 585 (67\%) were excluded and 288 children were randomized to either ASAQ or AL. At day 42 of follow up, the treatment efficacy of ASAQ was $78 \%$ before and $95 \%$ after PCR correction for reinfections. In the AL-arm, treatment efficacy was $84 \%$ before and $99.0 \%$ after PCR correction. Treatment efficacy after PCR correction was within the margin of non-inferiority as set for this study. Fewer children in the AL arm reported adverse reactions.

Conclusions: ASAQ is still effective as a treatment for uncomplicated malaria in Baraka, South Kivu, DRC. In this region, AL may have higher efficacy but additional trials are required to draw this conclusion with confidence. The high re-infection rate in South-Kivu indicates intense malaria transmission.

Trial registration NCT02741024

Keywords: Uncomplicated malaria, Artesunate-amodiaquine, Artemether-lumefantrine, Child, Treatment efficacy, DR Congo, Plasmodium falciparum, DRC

\footnotetext{
*Correspondence: marit.de.wit@amsterdam.msf.org

${ }^{1}$ Médecins sans Frontières (MSF), Plantage Middenlaan 14, 1018

DD Amsterdam, The Netherlands

Full list of author information is available at the end of the article
} 


\section{Background}

The Democratic Republic of Congo (DRC) is one of the five countries with the highest malaria burden in the world [1]. The eastern part of DRC is suffering from armed conflict and internal political instability. The conflict has caused massive suffering for civilians, with estimates of millions of deaths, directly or indirectly, as a result of the fighting $[2,3]$. In this humanitarian crisis there are acute health needs, with limited access to humanitarian assistance and violations of basic rights and freedoms. A survey conducted in 2014 in South Kivu indicated a crude mortality rate (CMR) of 2.80 per 10,000 per day [ $95 \% \mathrm{CI}(2.40-3.28)]$ and an under-five mortality rate $(<5 \mathrm{MR})$ of 5.58 per 10,000 per day [95 \% CI $(4.60-$ 6.76)]. Both of these mortality rates are more than two times above the emergency threshold [4].

Médecins sans Frontières-Operational Centre Amsterdam (MSF-OCA or MSF) has been working in the provinces of North Kivu and South Kivu since the early 1990s and in Katanga since 2003 (Fig. 1). In South Kivu, MSF supports primary and secondary health care in the Baraka and Kimbi hospitals and in six health centres, and provides response to outbreaks and emergencies.
Malaria is holo-endemic in most of DRC, with seasonal fluctuations in transmission intensity in the east and south of the country. Plasmodium falciparum is the predominant species causing malaria in DRC [5]. Since 2003, MSF has introduced artemisinin-based combination therapy (ACT) in all programmes worldwide. Artesunate-amodiaquine (ASAQ) has been the first-line treatment for uncomplicated malaria in DRC since 2005. Artemether-lumefantrine (AL) is the second line treatment for uncomplicated malaria $[6$, 7]. Targeted distribution of long-lasting insecticide-treated bed nets (LLIN) to pregnant women and children hospitalized for severe malaria has been ongoing since 2003 in the MSF Baraka programme. The last mass LLIN distribution in this health zone was carried out in 2012.

Since 2009, MSF has seen an apparent rise in malaria incidence in most programmes in DRC. The National Malaria Control Programme (Programme National de Lutte contre le Paludisme, PNLP) has also observed an increase in malaria cases. In the Baraka programme, without any significant changes to the programme activities, the number of parasitologically confirmed uncomplicated malaria cases has risen from 7457 in 2009 to 44,317 cases in 2012 (Fig. 2). In order to investigate whether the

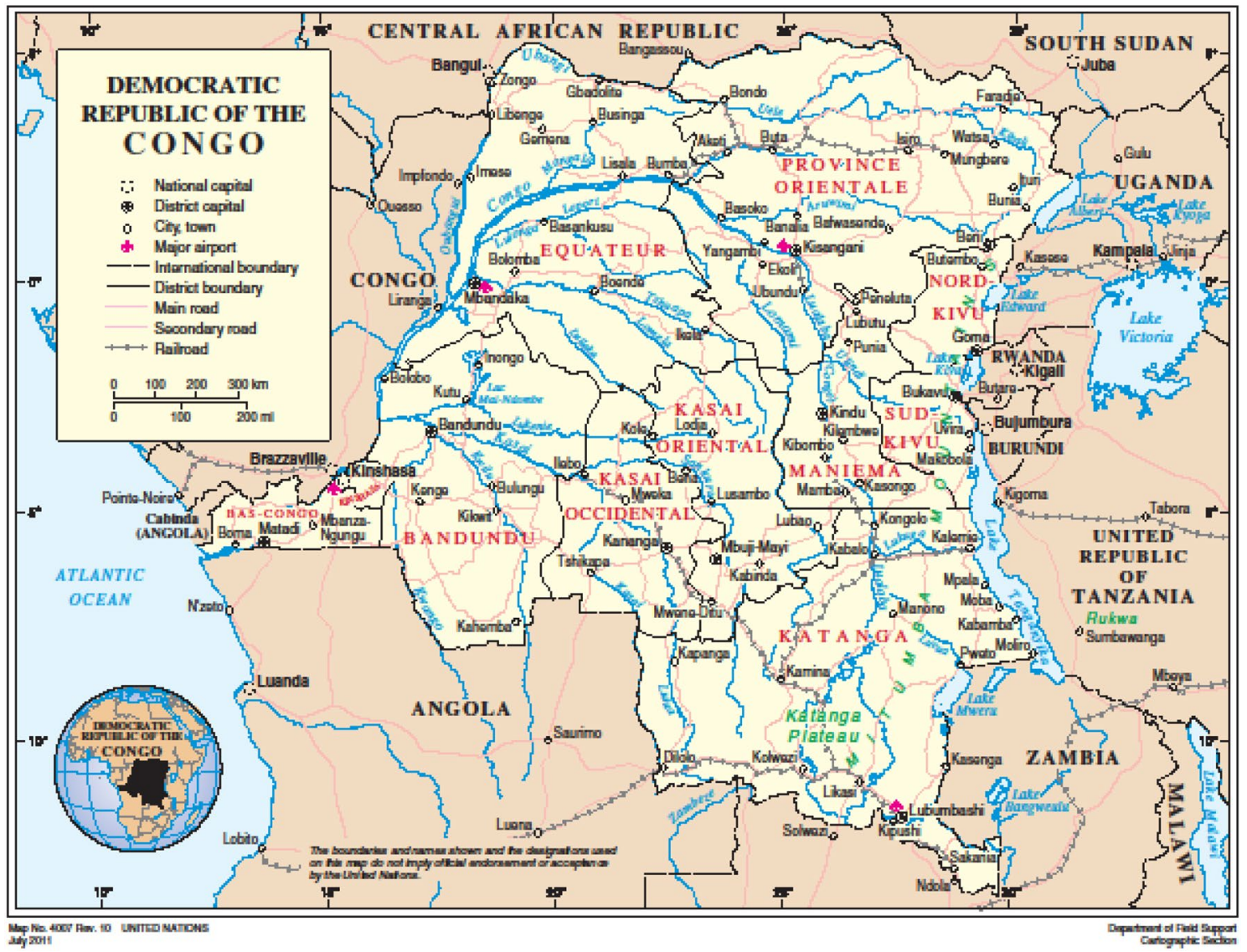

Fig. 1 Map of the Democratic Republic of Congo 


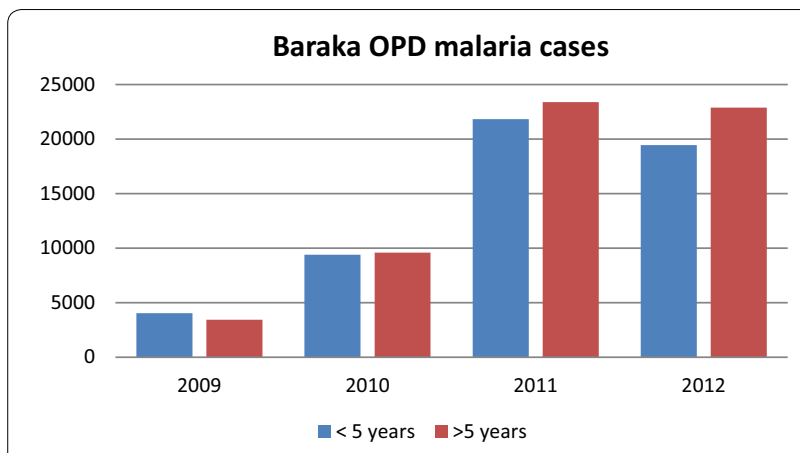

Fig. 2 Confirmed malaria cases in Baraka project 2009-2012, DRC

observed increase in malaria over the years was associated with increased treatment failure rates, the efficacy of the first-line treatment, ASAQ was evaluated, and compared to that of AL in children aged 6-59 months with confirmed uncomplicated falciparum malaria.

\section{Methods}

\section{Study design and site}

An open-randomized non-inferiority trial comparing the efficacy of ASAQ to that of AL for the treatment of uncomplicated malaria, in children aged between six and 59 months, was conducted following the World Health Organization (WHO) protocols for surveillance of antimalarial drug efficacy [8]. The study was carried out between October 2013 and December 2014 in the outpatient clinic of Baraka General Hospital and in the Health Centre of Baraka in South Kivu, DRC.

\section{Ethical considerations}

The study was approved by the Ethical Review Board (ERB) of MSF on June 17th 2013 and by the Zone Chief Medical Officer (Médecin Chef de Zone) and the Provincial Medical Inspector (Médecin Inspecteur Provençal MIP) of South Kivu (N251/969/B.MIP/SK/2013). It was registered at ClinicalTrials.gov under number NCT02741024. Eligible patients were included in the study after an adult parent/caretaker gave written informed consent. If the parent/caretaker was illiterate, a literate witness was asked to sign next to the parent/caretaker's fingerprint. Free health care for malaria and other illnesses is provided by MSF to the general population and was, therefore, also provided throughout the study follow-up period to all patients included in the study.

\section{Study population}

Children aged between 6 and 59 months, presenting with fever (axillary temperature $\geq 37.5{ }^{\circ} \mathrm{C}$ ) or reported history of fever in the last $24 \mathrm{~h}$, and with a positive rapid diagnostic test (RDT) were enrolled in the study if they had: (1) a body weight $\geq 5 \mathrm{~kg}$, (2) a slide confirmation of mono-infection with $P$. falciparum with an asexual parasite density between 2000 and 200,000/ $\mu$ l of blood, (3) an ability to swallow (crushed or dissolved) oral medication, (4) a high probability of respecting follow up visits, and (5) a signed informed consent by their adult ( $\geq 18$ years old) parent/caretaker. Children were excluded if they presented with general danger signs according to the WHO protocol "Methods for surveillance of antimalarial drug efficacy" [8]. These included signs of severe/ complicated malaria, including severe anaemia $(\mathrm{Hb}<5 \mathrm{~g} /$ $\mathrm{dL}$ ), history of convulsions, and jaundice. Children were also excluded if they had severe acute malnutrition (indicated by a weight-for-height $\mathrm{Z}$ score (WHZ) of $<-3 \mathrm{Z}$, a middle upper arm circumference (MUAC) of $<115 \mathrm{~cm}$, or bilateral oedema), a concomitant febrile or chronic illness, a known allergy to one of the study medications, or had received a full course of one of the artemisinin-based combinations under study in the previous 28 days.

\section{Sample size}

Sample size calculations were based on the data that was available before October 2012. At that moment, studies determined the 42-day risk of recurrent parasitaemia due to recrudescence (treatment failure) in children to range from 0.9 to $6 \%$ with AL and ASAQ [9-12]. Based on a conservative estimated risk of recurrent parasitaemia (due to recrudescence, PCR corrected) of $5 \%, 120$ patients per treatment arm would be needed to detect a difference in the risk of recrudescence between treatment arms of no greater than $7 \%$ (one-sided type I error of 5 , $80 \%$ power) [13]. In order to account for undetermined PCR results, loss to follow up, withdrawal, and protocol violations, the total estimated sample size was increased by $20 \%$ to 288 patients ( 144 per arm).

\section{Study procedure}

All patients who were eligible for the study had a medical examination, and a MUAC and WHZ screening. A thick and a thin blood smear were performed and capillary blood was collected on a fast technology for analysis (FTA) card (Whatmann, UK). If the patient was eligible, informed consent was requested from the parent/caretaker after the explanation of the study by one of the study team members. Once the informed consent was obtained, patients were randomized to one of two treatment regimens according to which treatment had been randomly pre-allocated to that unique patient number. Treatment regimens consisted of: (1) ASAQ fixed dose (Winthrop Sanofi Aventis), given as 1 tab/day over three days $(\geq 5-<9 \mathrm{~kg}, 1 \mathrm{tab}$ of $25 \mathrm{mg}$ artesunate/67.5 $\mathrm{mg}$ 
amodiaquine base; $\geq 9-<18 \mathrm{~kg}, 50 \mathrm{mg}$ artesunate/135 mg amodiaquine base), or (2) AL fixed combination (Coartem $^{\circledR}, 20 \mathrm{mg}$ artemether $/ 120 \mathrm{mg}$ lumefantrine, Novartis) given with milk twice daily over three days $(\geq 5-<15 \mathrm{~kg}, 1$ tab of $20 \mathrm{mg}$ artemether $/ 120 \mathrm{mg}$ lumefantrine BD, $\geq 15-$ $<25 \mathrm{~kg}, 2$ tabs of $20 \mathrm{mg}$ artemether/120 mg lumefantrine BD with fatty food) over 3 days. Drugs were given according to the manufacturer's instructions and dosage. Study medication was imported and stored under strict regulations, ensuring control within the provided storage temperature and humidity ranges. The first dose of the study drug at day 0 (D0) was dispensed at the health clinic, and was taken by the child while under supervision of a study team member. For children who could not swallow tablets, AL tablets were dissolved, and ASAQ tablets were crushed in minimal amounts of water and dispensed with a spoon. After intake, the child was observed for $30 \mathrm{~min}$. If the child vomited or spat out the medication within the monitoring period, a resting period of $15 \mathrm{~min}$ was observed before re-administering a repeat dose. If the repeat dose was also vomited within $30 \mathrm{~min}$, the child was administered a rescue treatment (which was defined as the alternative treatment that the child was not randomized to) and was excluded from the study. Patients returned to the clinic on days 1 and 2 for observed administration of the study drug and a clinical assessment. In order to observe the intake of the evening doses of AL, patients randomized to receive AL were visited at home at around $8 \mathrm{~h}, 32 \mathrm{~h}$ and $56 \mathrm{~h}$ after the first dose. A follow up was scheduled for all patients on day $3,7,14,21$, 28, 35 and 42 for clinical assessment and blood smear evaluation.

If a clinical or parasitological treatment failure was observed, rescue treatment was initiated and a second blood sample was collected for PCR analysis. Caretakers were advised to present the child to the clinic at any time in case of illness. Patients who failed to return for scheduled follow up visits were traced immediately by home-visitors to minimize loss to follow up. Adverse events were evaluated by a physician and recorded at each visit.

\section{Treatment outcomes}

Study endpoints were classified according to the WHO guidelines [8] as adequate clinical and parasitological response (ACPR), early treatment failure (ETF), late clinical failure (LCF) and late parasitological failure (LPF). Patients who were lost to follow-up, who withdrew their consent at any time before reaching a study endpoint, or who had a protocol violation (ex. wrongful inclusion by study staff, non-completion of full treatment course, administration of a study drug by a third-party, re-infection with a species other than $P$. falciparum) were not assigned an efficacy treatment outcome.

\section{Laboratory techniques}

Initial testing for malaria infection was done on finger-prick blood using an RDT, the SD Bioline 05FK50 Malaria Ag P.f (Standard Diagnostics, Kyonggi, Republic of Korea). This RDT relies on detection of the Histidine Rich Protein 2 (HRP2), and is amongst the most sensitive according to the WHO product testing programme [14].

Thick and thin blood smears were stained with $10 \%$ Giemsa for $15 \mathrm{~min}$. Smears were read to 100 fields before they could be declared negative. Species were confirmed on the thin smear. Quantification of P. falciparum asexual parasitaemia on the thick smear was performed according to the WHO protocol "Methods for surveillance of antimalarial drug efficacy" [8]. Presence or absence of $P$. falciparum gametocytes was recorded.

Each slide was read, independently, by two qualified microscopists in the Baraka hospital laboratory, Parasite densities were calculated by taking a mean of the two counts. Blood smears with discordant results (differences in species diagnosis, in parasite density of $>50 \%$ or in the presence of parasites) were re-examined by a third microscopist who was blinded to the results of the first two, and parasite density was calculated by taking a mean of the two closest counts. In addition, external quality control of the slides was performed by blinded re-checking of 50 randomly selected malaria slides by an expert microscopist at the Epicentre Mbarare Research Base in Uganda.

Haemoglobin levels on D0 or during follow-up were determined using the HemoCue ${ }^{\circledR} \mathrm{Hb} 301$ System (Ängelholm, Sweden), according to manufacturer's instructions. PCR genotyping analysis was performed in order to distinguish true recrudescence (same parasite strain) from a newly acquired infection (different parasite strain) on capillary blood samples stored on FTA cards. The genotyping was performed at the Department of Medical Microbiology, at the Academic Medical Centre (Amsterdam, The Netherlands. PCR amplification of template DNA and analysis of glurp, msp 2 and msp 1 alleles in pre(enrolment) and post-treatment (failure) samples was performed according to the WHO protocol [15]. Pre- and post-treatment pairs with similar genotype were classified as recrudescence (true failure), and pairs with different genotypes were classified as re-infection according to WHO protocol "Methods and techniques for clinical trials on antimalarial drug efficacy: Genotyping to identify parasite populations" [15]. Species-specific PCRs were performed on samples with possible mixed re-infections according to Shokoples et al. [16]. 


\section{Data analysis}

All data were double entered in the WHO global database for anti-malarial drug efficacy by two different epidemiologists, as well as in the worldwide anti-malarial resistance network (WWARN) database provided online [17]. Statistical analysis was performed using STATA version 13.1. Data was analysed per-protocol and by intentionto-treat. Kaplan-Meier survival curves were generated to determine the cumulative probability of recurrence-free survival over the 42 days follow-up. These probability estimates were compared using a log rank test. For the per-protocol analysis, patients who were lost to follow up or withdrawn were removed from the denominator. Patients were considered withdrawn from the PCR-corrected analysis if the PCR results were unclassifiable or if the results of PCR indicated that the failure was due to a mono re-infection with a Plasmodium species other than the P. falciparum (such as Plasmodium vivax, Plasmodium malariae or Plasmodium ovale).

Chi square tests or Fisher's exact tests were used to compare categorical data and Student's $t$ test was used for continuous data, as appropriate. The log rank test was used to compare Kaplan-Meier survival curves. p $<0.05$ were considered statistically significant.

\section{Availability of data and materials}

Data are available on request in accordance with MSF's data sharing policy [18].

\section{Results}

\section{Study trial profile and baseline characteristics}

A description of the study outline is shown in Fig. 3. Out of 873 children screened for uncomplicated malaria during the study period, 585 (67.0\%) were excluded. The main reasons for exclusion were ACT intake in the last 28 days $(118,20.2 \%)$, parasitaemia outside the study range $(116,19.8 \%)$ or co-morbidities such as pneumonia or urinary tract infections $(86,14.7 \%) .288$ children were included in the study and were randomized to receive ASAQ or AL. 18 children (6.3\%) in the ASAQ group and 13 children (4.5\%) in the AL group did not complete the three days of allocated treatment, due to the following: developing (apparent) signs of severe malaria ( $n=5 \mathrm{ETF}$, $\mathrm{n}=3$ wrongfully admitted to ward), a late realisation of wrongful enrolment $(n=10)$, having an observed treatment dose missed $(n=7)$, vomiting of at least one of the treatment doses twice $(n=5)$, a dosage error $(n=1)$. Of the children who received their full course of supervised treatment (257), 19 children $(7.0 \%)$ did not reach an analysable end result (censored), either due to being lost to follow up ( $\mathrm{n}=8)$, having had an anti-malarial administered by a $3^{\text {rd }}$ party $(n=9)$, or having been re-infected with a malaria species other than P. falciparum $(n=2)$.
The baseline characteristics of the patients enrolled in the study are presented in Table 1 . Boys and girls were equally represented in the study population, with an average age of 31.5 months [(32.2 months for ASAQ (IQR 20.0-47.0) and 30.9 for AL (IQR 21.0-41.5)]. The average WHZ score was below international standards and similar in both treatment groups ( -0.59 for ASAQ and -0.68 for AL) [19]. The average axillary temperature at D0 was higher in the AL group $\left(39.02{ }^{\circ} \mathrm{C}\right.$, IQR 38.0-39.7) than in the ASAQ group $\left(38.77^{\circ} \mathrm{C}\right.$, IQR $\left.38.4-39.8\right)$. No other statistically significant differences were observed between baseline characteristics for children randomized in both treatment arms.

\section{Treatment outcome results}

Five children were classified as early treatment failures as per study protocol. All of these children had a parasitological improvement, but had either anaemia or jaundice that warranted admission to the hospital. They received intravenous artesunate according to the study protocol and the WHO guidelines for the treatment of malaria [20] and were withdrawn from the study.

Treatment efficacy estimates are presented in Table 2 for days 28 and day 42 after initiation of treatment. Before PCR genotyping, 23 (9.7\%) children (13/119 on ASAQ and $10 / 119$ on $\mathrm{AL}$ ) were classified as late clinical failure and $23(9.7 \%)$ children (14/119 on ASAQ and 9/119 on $\mathrm{AL})$ as late parasitological failure on day 42. Furthermore, during the study period, two children who were on AL were re-infected with other species ( $P$. ovale), as confirmed in weekly parasitological examinations; this was an involuntary protocol violation and these two children were censored in the final analysis. In total, 192 (80 \%) children had an adequate clinical and parasitological response (92/119 for ASAQ and 100/119 for AL) at day 42. The proportion of recurring infection (including the two reinfections with other species) during the 42 days follow-up was higher in the ASAQ group (27/119, $22.7 \%$ ) compared to the AL group (21/119, $17.6 \%)$, but this difference was not statistically significant. Similarly, cumulative failure on day 28 was not statistically significantly different between arms.

\section{PCR genotyping to determine re-infection and recrudescence}

46 paired FTA filter paper samples were PCR genotyped, including all late clinical and parasitological failure samples. Following the PCR genotyping, 21 of the 27 recurrent parasitaemic episodes in the ASAQ arm (77.8\%) were classified as re-infections and $6(22.2 \%)$ as recrudescence. In the AL arm, 15 out of 19 samples (78.9\%) were classified as re-infections and 1 as (5.3\%) as recrudescence. There were no indications that treatment 
Trial profile

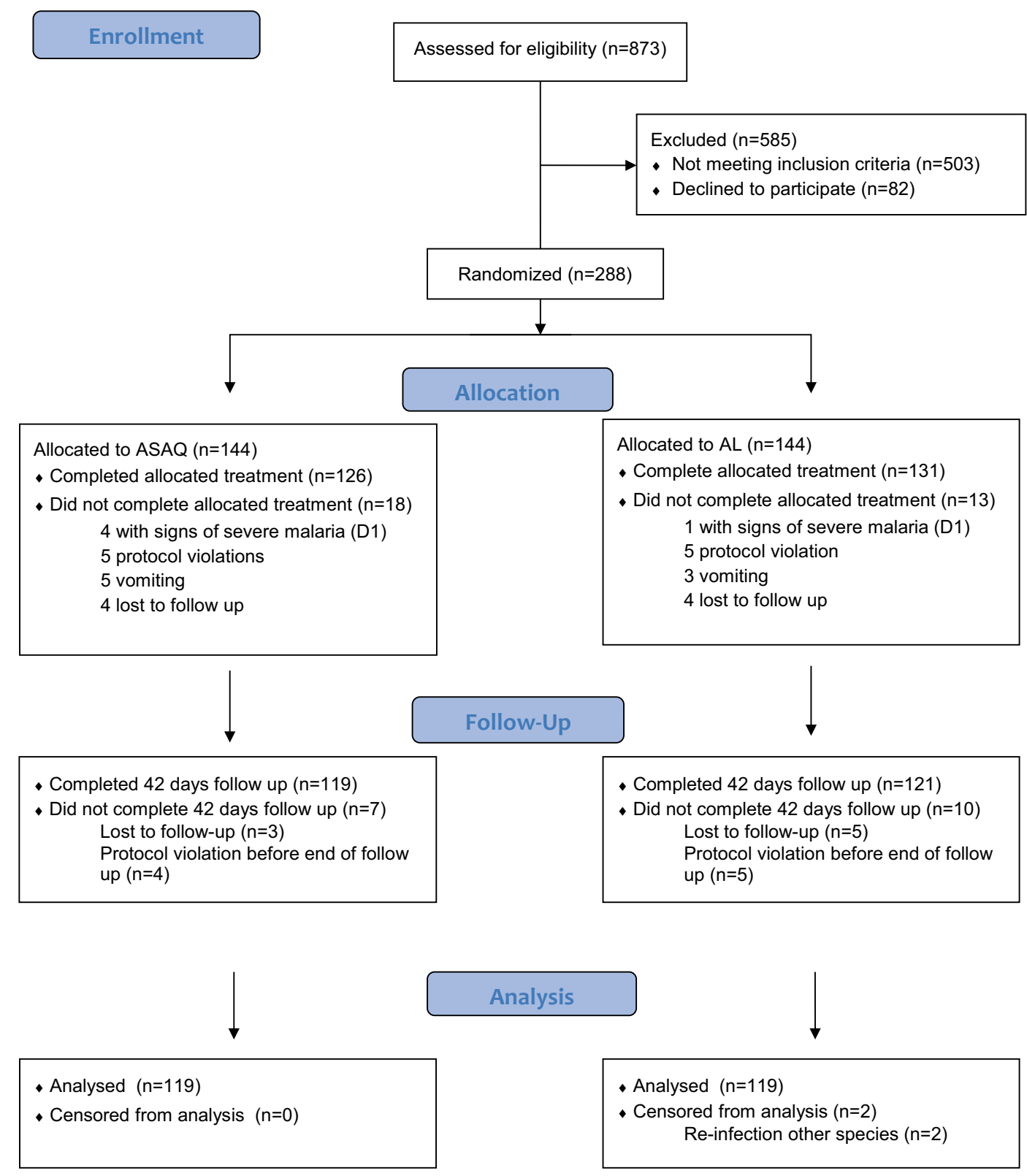

Fig. 3 Trial profile efficacy study ASAQ-AL, Baraka, DRC

dosing was lower for the children experiencing treatment failure.

For three samples with recurring parasitaemia in the AL arm outcome could not be classified by PCR; these samples were censored at the last date of follow-up for which the child was still malaria negative. The proportions of children reaching each a study endpoint, before and after PCR adjustment, are presented in Table 2.

\section{Kaplan-Meier survival curves}

The cumulative probability of recurrence-free survival over the 42 days follow-up was determined by KaplanMeier survival analysis. The PCR un-adjusted curves shown in Fig. 4 indicate a non-significantly higher probability of remaining free of recurrent parasitaemia in the $\mathrm{AL}$ arm compared to the ASAQ arm $(\mathrm{p}=0.16)$. After adjustment by PCR to distinguish between reinfection 
Table 1 Baseline characteristics of included patients

\begin{tabular}{|c|c|c|c|}
\hline & ASAQ $(n=144)$ & $A L(n=144)$ & p value \\
\hline Age, in months & $32.2(20.0-47.0)$ & $30.9(21.0-41.5)$ & 0.412 \\
\hline \# Male (\%) & $65(79)$ & $65(79)$ & 1.000 \\
\hline Weight for height Z-score & $-0.59(-1.39$ to 0.22$)$ & $-0.68(-1.40-0.08)$ & 0.484 \\
\hline Temperature in ${ }^{\circ} \mathrm{C}$ & $38.8(38.0-39.7)$ & $39.02(38.4-39.8)$ & 0.049 \\
\hline Parasite density/ $\mu$ lat day 0 & $63,637(15,211-95,962)$ & $45,154(14,225-94,746)$ & 0.826 \\
\hline Log parasite density at day 0 & $10.5(9.6-11.5)$ & $10.4(9.6-11.5)$ & 0.935 \\
\hline Haemoglobin in $\mathrm{g} / \mathrm{dl}$ at day 0 & $9.5(8.5-10.8)$ & $9.7(8.7-10.8)$ & 0.316 \\
\hline
\end{tabular}

All data is presented as the mean with the interquartile range (IQR) in brackets, unless otherwise stated

Table 2 Per-protocol crude/unadjusted and PCR-adjusted study endpoints at days 28 and 42 of patient follow-up. Patients with re-infections and individuals for whom the outcome could not be assessed by PCR were censored from the PCR adjusted analysis

\begin{tabular}{|c|c|c|c|c|c|c|c|c|c|c|}
\hline \multirow[t]{3}{*}{ Crude/no PCR } & \multicolumn{5}{|c|}{ Day 28} & \multicolumn{5}{|c|}{ Day 42} \\
\hline & \multicolumn{2}{|c|}{ ASAQ $(n=119)$} & \multicolumn{2}{|c|}{$A L(n=122)$} & \multirow[t]{2}{*}{$p$ value } & \multicolumn{2}{|c|}{$\begin{array}{l}\text { ASAQ } \\
(n=119)\end{array}$} & \multicolumn{2}{|c|}{$\mathrm{AL}(\mathrm{n}=119)$} & \multirow[t]{2}{*}{ p value } \\
\hline & $\mathrm{n}$ & $\%$ & $\mathbf{n}$ & $\%$ & & $\mathbf{n}$ & $\%$ & $\mathbf{n}$ & $\%$ & \\
\hline Late clinical failure & 8 & 6.7 & 4 & 3.3 & 0.250 & 13 & 10.9 & 10 & 8.4 & 0.510 \\
\hline Late parasitological failure & 10 & 8.4 & 6 & 4.9 & 0.277 & 14 & 11.8 & 9 & 7.6 & 0.273 \\
\hline Adequate clinical and parasitological response & 101 & 84.9 & 112 & 91.8 & 0.093 & 92 & 77.3 & 100 & 84.0 & 0.189 \\
\hline Cumulative failure & 18 & 15.1 & 10 & 8.2 & 0.093 & 27 & 22.7 & 19 & 16.0 & 0.189 \\
\hline \multirow[t]{3}{*}{ PCR adjusted/corrected } & \multicolumn{5}{|c|}{ Day 28} & \multicolumn{5}{|c|}{ Day 42} \\
\hline & \multicolumn{2}{|c|}{ ASAQ $(n=105)$} & \multicolumn{2}{|c|}{$A L(n=111)$} & \multirow[t]{2}{*}{$\mathrm{p}$ value } & \multicolumn{2}{|c|}{ ASAQ $(n=98)$} & \multicolumn{2}{|c|}{$A L(n=101)$} & \multirow[t]{2}{*}{$\mathrm{p}$ value } \\
\hline & $\mathbf{n}$ & $\%$ & $\mathrm{n}$ & $\%$ & & $\mathbf{n}$ & $\%$ & $\mathrm{n}$ & $\%$ & \\
\hline Late clinical failure (recrudescence) & 1 & 1.0 & 0 & 0 & 0.486 & 1 & 1.0 & 1 & 1.0 & 1.000 \\
\hline Late parasitological failure (recrudenscence) & 3 & 2.9 & 0 & 0 & 0.113 & 5 & 5.1 & 0 & 0 & 0.027 \\
\hline Adequate clinical and parasitological response & 101 & 96.2 & 111 & 100 & 0.038 & 92 & 93.9 & 100 & 99.0 & 0.049 \\
\hline Cumulative failure & 4 & 3.8 & 0 & 0 & 0.054 & 6 & 6.1 & 1 & 1.0 & 0.062 \\
\hline
\end{tabular}

The analysis of the treatment outcomes by intention-to-treat are shown in Additional file 1: Annexure 1

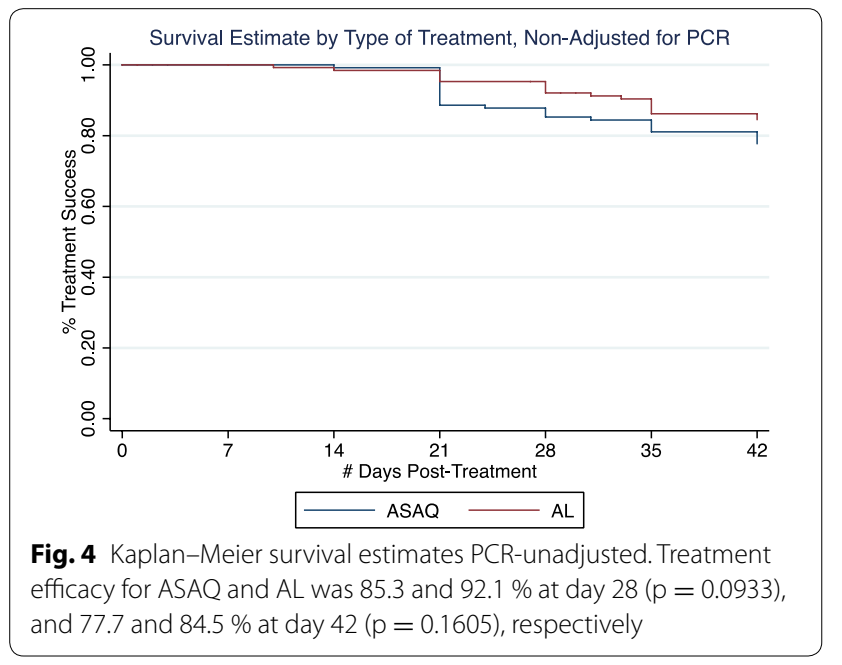

and recrudescence, the proportion of patients achieving ACPR at day 42 (treatment efficacy) was higher for the AL arm (99.0\%, 95CI 93.2-99.9) compared to the ASAQ arm (94.7 \%, 95 CI 88.4-97.6), $\mathrm{p}=0.0496$ (Fig. 5). Regardless of the statistically significant differences between study arms, the difference in ACPR by day 42 after initiation of treatment was within the margin of non-inferiority.

\section{Parasite clearance}

A relatively large proportion (13.7 \% on ASAQ, $20.0 \%$ on $\mathrm{AL}$ ) of children still had parasites on day 2 of follow-up. This proportion fell to below $5 \%$ in both arms on day 3 . There was no significant difference in the proportion of parasites at D2 and D3 between the two treatment arms (Table 3). 


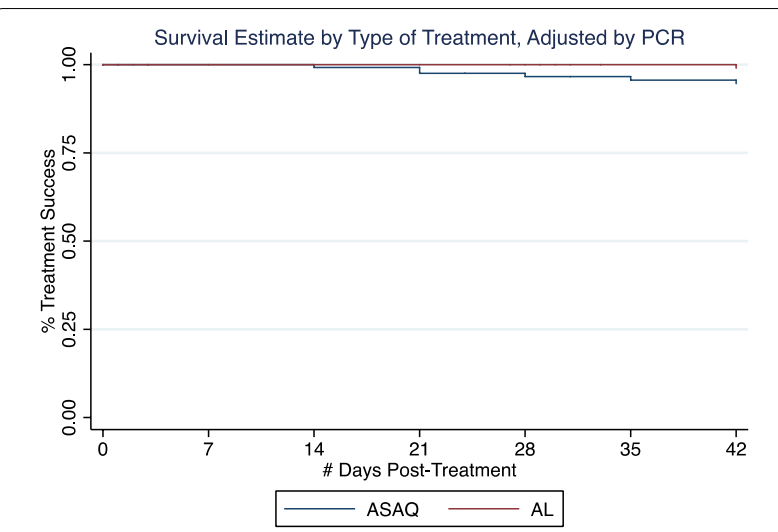

Fig. 5 Kaplan-Meier survival estimates PCR-adjusted. Treatment efficacy for ASAQ and AL was 96.6 and $100 \%$ at day 28 ( $p=0.0406$ ), and 94.7 and $99.0 \%$ at day $42(p=0.0496)$, respectively

Table 3 Parasite clearance on Days 2 and 3 of treatment

\begin{tabular}{llll}
\hline & ASAQ & AL & p value \\
\hline $\begin{array}{l}\text { Patients with parasite } \\
\text { positivity at D2 }\end{array}$ & $18(13.7 \%)$ & $27(20.0 \%)$ & 0.173 \\
$\begin{array}{l}\text { Patients with parasite } \\
\text { positivity at D3 }\end{array}$ & $2(1.5 \%)$ & $6(4.4 \%)$ & 0.167 \\
\hline
\end{tabular}

\section{Treatment safety}

There were no serious adverse events during the period of the study. However, minor adverse events occurred in both treatment arms during the treatment period (days $0-2)$. The adverse event most reported was asthenia (weakness, lack of energy and strength) for both arms. Children receiving ASAQ reported significantly more asthenia and anorexia (loss of appetite) than children receiving AL (Table 4).

Table 4 Adverse events = classified as related to the drug, for all patients during the study period

\begin{tabular}{llllr}
\hline & $\begin{array}{l}\text { ASAQ } \\
(\mathbf{n}=\mathbf{1 4 4 )}\end{array}$ & AL $(\mathbf{n}=\mathbf{1 4 4})$ & Total & p value \\
\hline Asthenia & $61(42.4 \%)$ & $13(9.0 \%)$ & $74(25.7 \%)$ & $<0.001$ \\
Anorexia & $27(18.8 \%)$ & $12(8.3 \%)$ & $39(13.5 \%)$ & 0.010 \\
Vomiting & $11(7.6 \%)$ & $10(6.9 \%)$ & $21(7.3 \%)$ & 0.821 \\
Cough & $5(3.5 \%)$ & $7(4.9 \%)$ & $12(4.2 \%)$ & 0.555 \\
Abdominal & $6(4.2 \%)$ & $2(1.4 \%)$ & $8(2.8 \%)$ & 0.151 \\
$\quad$ Pain & & & & \\
Diarrhoea & $4(2.8 \%)$ & $2(1.4 \%)$ & $6(2.1 \%)$ & 0.409 \\
Itching & $3(2.1 \%)$ & $1(0.7 \%)$ & $4(1.4 \%)$ & 0.314 \\
Nausea & $1(0.7 \%)$ & $1(0.7 \%)$ & $2(0.7 \%)$ & 1.000 \\
\hline
\end{tabular}

\section{Discussion}

The results of this study indicate that both study drugs retain adequate efficacy (PCR corrected cure rate at day 42 of $94.7 \%$ for ASAQ and $99.0 \%$ for AL) in the treatment of uncomplicated $P$. falciparum malaria in children aged six to 59 months in the study setting. In 2004, a similar study in DRC (Pool Region) showed comparable results (98.5\% efficacy of ASAQ and $100 \%$ efficacy of AL at 28 days) [21]. This was also the result of the efficacy study by Singana et al. [22] in Owando in Congo-Brazzaville done in 2012-2103 (efficacy of ASAQ $98.0 \%$ and of AL $100.0 \%$ at 28 days, after PCR correction). The $4 \mathrm{ABC}$ study group, performed in a comparable population and using the same methodology, showed a similar efficacy rate between 2007 and 2009 over seven sub-Saharan African countries (96.8\% efficacy of ASAQ and $95.5 \%$ efficacy of AL at 28 days) [23]. In the $4 A B C$ study, ASAQ was also shown to be effective in Eastern Africa, whereas other studies had previously casted doubts over its efficacy in this region [24, 25].

The high efficacy of both ASAQ and AL in this region is reassuring. WHO only recommends a switch in the first line treatment if the efficacy falls below $90 \%$ [26]. Although this study confirms that both artemisininbased combinations retain good efficacy in this part of DRC, it does, however, demonstrate high re-infection levels. After PCR correction, this study showed that, over 6 weeks, $21 / 119$ children $(17.6 \%)$ of those who were on ASAQ and 15/119 (12.6\%) of the children in the AL treatment group $(\mathrm{p}=0.29)$ were re-infected within 42 days.

Whilst re-infections do not indicate failing anti-malarial drugs, they are of public health relevance and pose a considerable burden to the population and health systems. The results of this study seem to support recent findings that the period over which lumefantrine and amodiaquine provide post-treatment protection is 10-14 days [26-28]. However, considering the public health impact of frequent malaria infections in infants, it would be useful to do further analysis into the specific protective period for each of ASAQ and AL in this setting. It is also worth considering if the use of an ACT with a longer protective half-life, such as dihydroartemisininpiperaquine, may be beneficial to prevent malaria episodes in the study population, exposed to intense malaria transmission [27-29].

In this study's setting children will normally receive ASAQ each time they have a malaria infection. Reassuringly, Yeka et al. [29] showed that repeated treatment using both ASAQ and AL is effective, safe and well-tolerated in children under 5 years of age and in a similar context [29]. 
With regards to tolerability, there were no serious adverse events registered during the trial. Asthenia and anorexia were the most commonly recorded minor adverse events with a marked difference between the two drugs. It was shown by the results of this study that AL was better tolerated. During the study it was observed that the parents/caretakers would prefer that their child be randomized to AL rather than ASAQ as the former was perceived as more tolerable by the children. In fact, the chance to be randomized to receive AL seemed to be a major driving force to participate in the study. Although asthenia and anorexia may be classified as minor adverse effects, it can have a negative effect on adherence, and thus prevent a complete cure.

There was a relatively high proportion $(67 \%)$ of children excluded from the study at the time of screening. There were two major reasons for exclusion: the high number of children with co-morbidities, and the high number of children who had taken ACT in the previous 28 days (either from a clinic or from a private vendor). In the children with co-morbidities, it is not clear what relation malaria had to their clinical illness; the children could be presenting with clinical illness due to malaria, or have symptoms primarily from their co-morbidity but happen to have malaria parasites. This is a dilemma that is faced often in the field, and may lead to an overestimation of the burden of malaria. Furthermore, an RDT for malaria is often performed as a first means of triage for fever, and when the test is positive, there is the risk that the child may not be adequately examined for other illnesses. In this study, 49 of the 873 (5.6\%) children with a positive RDT (based on HRP2 detection) had a negative thick smear. This could be the result of circulating HRP2 from a previous, well treated malaria infection. This is expected considering that Grandesso et al. (internal communication, Clinical Trial. gov Identifier NCT01325974) have shown that over $50 \%$ of HRP2 RDTs remain positive more than 6 weeks after adequate treatment of a malaria infection.

\section{Conclusion}

ASAQ is still effective as a treatment for uncomplicated malaria in Baraka, South Kivu, DRC, 9 years after its introduction as a first-line treatment. There is no evidence that ASAQ is inferior to AL in this setting with the non-inferiority margin of $7 \%$ that was set for the current study. AL may have higher efficacy but additional trials are required to draw this conclusion with confidence. There is a high rate of re-infection in South-Kivu within six weeks of adequate treatment of either ASAQ or AL, indicating intense malaria transmission. AL was better tolerated by patients than ASAQ. Although earlier estimates of anti-malarial drug efficacy are unavailable for the current population, the high efficacy of ASAQ makes it unlikely that declining drug efficacy is a reason for the apparent increase in malaria cases observed in the region.

\section{Additional file}

Additional file 1: Annexure 1. Intent-to-treat crude/unadjusted and PCR-adjusted study endpoints at days 28 and 42 of patient follow-up.

\begin{abstract}
Authors' contributions
$\mathrm{RS}, \mathrm{KB}$ and MdW jointly drafted the proposal and supported the study. KM set-up and implemented the study in the field as lead field epidemiologist. $\mathrm{KM}$ and AF coordinated the field component of the study as field epidemiologists. EP and $A B$ gave input to and supervised the laboratory component. DAN was one of the study clinic supervisors. BB reviewed the proposal and the article. AF and MdW performed statistical analysis. MdW was the principal investigator and wrote the article. TB contributed to the statistical analysis and supervised the analysis of the study and the article. All authors reviewed and approved the article. All authors read and approved the final manuscript.
\end{abstract}

\section{Author details}

${ }^{1}$ Médecins sans Frontières (MSF), Plantage Middenlaan 14, 1018 DD Amsterdam, The Netherlands. ${ }^{2}$ Manson Unit, Médecins Sans Frontières (MSF), 10 Furnival Street, London EC4A 1AB, UK. ${ }^{3}$ Academisch Medisch Centrum, Amsterdam, The Netherlands. ${ }^{4}$ Programme National de lutte contre le Paludisme, Kinshasa, South Kivu, Democratic Republic of the Congo. ${ }^{5}$ London School of Hygiene and Tropical Medicine, London, UK. ${ }^{6}$ Radboud university medical center, Nijmegen, The Netherlands.

\section{Acknowledgements}

The study team would like to thank the South Kivu coordination team and the Baraka field team (Joe Wazome, David Aksanti Nkuba, Alain Asukulu Pelochose, Claude Bwende Habinamwisho, Patrick Bujingo Murhula, Christian Bwira Buciza, Edwige Hali Zinduna, Jean-Jacques Mapenzi Ndahe, Jonathan Nusi Makyata Ndale, Henri Mwessa Ngumba, Franck Okundji Lomami, Kester Elembe Baruani, Sébastien Akilimali Mukandama, Olivier Situmani Ngungumazi) and all the young study participants and their caretakers. Many thanks to Nienke Verhaar for her practical work in the AMC lab.

\section{Competing interests}

Thr authors declare that they have no competing interests.

Availability of data and material

Data are available on request in accordance with MSF's data sharing policy at http://www.fieldresearch.msf.org/msf/handle/10144/306488.

\section{Ethics approval and consent to participate}

The study was approved by the Ethical Review Board (ERB) of MSF on June $17^{\text {th }} 2013$ and by the Zone Chief Medical Officer (Médecin Chef de Zone) and the Provincial Medical Inspector (Médecin Inspecteur Provençal MIP) of South Kivu (N251/969/B.MIP/SK/2013). It was registered at ClinicalTrials.gov under number NCT02741024. Eligible patients were included in the study after an adult par-ent/caretaker gave written informed consent. If the parent/caretaker was illiterate, a literate witness was asked to sign next to the parent/caretaker's fingerprint. Free health care for malaria and other illnesses is provided by MSF to the general population and was therefore also provided throughout the study follow-up period to all patients included in the study.

\section{Funding}

The study was funded by MSF-OCA.

Received: 20 April 2016 Accepted: 18 July 2016

Published online: 06 September 2016 


\section{References}

1. WHO. World malaria report 2013. Geneva: World Heath Organization; 2013. p. 256.

2. Coghlan B, Brennan RJ, Ngoy P, Dofara D, Otto B, Clements M, et al. Mortality in the Democratic Republic of Congo: a nationwide survey. Lancet. 2006;367:44-51.

3. Coghlan B, Ngoy P, Mulumba F, Hardy C, Bemo VN, Stewart T, et al. Update on mortality in the Democratic Republic of Congo: results from a third nationwide survey. Disaster Med Public Health Prep. 2009;3:88-96.

4. Lenglet A. Retrospective Mortality Survey in the MSF Catchment Area in Fizi Health Zone, South Kivu. Amsterdam: Democratic Republic of Congo; 2014

5. WHO. Malaria Country Profile DRC. Geneva: World Heath Organization; 2014

6. PNLP DRC. Rapport annuel des activites de lutte contre le paludisme 2013. 2014

7. Ministère de la Santé DRC. Profil pharmaceutique de la République Démocratique du Congo. 2011.

8. WHO. Methods for surveillance of antimalarial drug efficacy. Geneva: World Heath Organization; 2009.

9. Mårtensson A, Strömberg J, Sisowath C, Msellem MI, Gil JP, Montgomery SM, et al. Efficacy of artesunate plus amodiaquine versus that of artemether-lumefantrine for the treatment of uncomplicated childhood Plasmodium falciparum malaria in Zanzibar, Tanzania. Clin Infect Dis. 2005:41:1079-86.

10. Espié E, Lima A, Atua B, Dhorda M, Flévaud L, Sompwe EM, et al. Efficacy of fixed-dose combination artesunate-amodiaquine versus artemetherlumefantrine for uncomplicated childhood Plasmodium falciparum malaria in Democratic Republic of Congo: a randomized non-inferiority trial. Malar J. 2012;11:174.

11. Yeka A, Dorsey G, Kamya MR, Talisuna A, Lugemwa M, Rwakimari JB, et al. Artemether-lumefantrine versus dihydroartemisinin-piperaquine for treating uncomplicated malaria: a randomized trial to guide policy in Uganda. PLOS ONE. 2008;3:e2390.

12. Zongo I, Dorsey G, Rouamba N, Dokomajilar C, Séré Y, Rosenthal PJ, et al. Randomized comparison of amodiaquine plus sulfadoxine-pyrimethamine, artemether-lumefantrine, and dihydroartemisinin-piperaquine for the treatment of uncomplicated Plasmodium falciparum malaria in Burkina Faso. Clin Infect Dis. 2007;45:1453-61.

13. Centre for Clinical Trials. Sample size calculator: two parallel-sample proportions. http://www.cct.cuhk.edu.hk/stat/proportion/tspp_sup.htm

14. WHO. Malaria rapid diagnostic test performance: results of WHO product testing of malaria RDTs: round 6 (2014-2015). Geneva: World Heath Organization.

15. WHO. MMV methods and techniques for clinical trials on antimalarial drug efficacy: genotyping to identify parasite populations. Geneva: World Heath Organization; 2007
16. Shokoples SE, Ndao M, Kowalewska-Grochowska K, Yanow SK. Multiplexed real-time PCR assay for discrimination of Plasmodium species with improved sensitivity for mixed infections. J Clin Microbiol. 2009;47:975-80.

17. Worldwide antimalarial resistance network toolkit. http://www.wwarn. org/tools-resources/toolkit/plan

18. MSF field research data sharing. http://fieldresearch.msf.org/msf/ handle/10144/306488

19. WHO. Weight-for-age. Geneva: World Heath Organization. http://www. who.int/childgrowth/standards/weight_for_age/en/

20. WHO. Guidelines for the treatment of malaria. 2nd ed. Geneva: World Heath Organization; 2010. p. 197.

21. Van den Broek I, Kitz C, Al Attas S, Libama F, Balasegaram M, Guthmann JP. Efficacy of three artemisinin combination therapies for the treatment of uncomplicated Plasmodium falciparum malaria in the Republic of Congo. Malar J. 2006:5:113.

22. Singana BP, Bogreau $H$, Matondo BD, Dossou-Yovo LR, Casimiro PN, Mbouka $R$, et al. Malaria burden and anti-malarial drug efficacy in Owando, northern Congo. Malar J. 2016;15:16

23. Group $4 A B C$. A head-to-head comparison of four artemisinin based combinations for treating uncomplicated malaria in African children: a randomized trial. PLoS Med. 2011;8:e1001119.

24. Clark TD, Njama-Meya D, Nzarubara B, Maiteki-Sebuguzi C, Greenhouse B, Staedke SG, et al. Incidence of malaria and efficacy of combination antimalarial therapies over 4 years in an urban cohort of Ugandan children. PLOS ONE. 2010;5:e11759.

25. Dorsey G, Staedke S, Clark TD, Njama-Meya D, Nzarubara B, Maiteki-Sebuguzi C, et al. Combination therapy for uncomplicated falciparum malaria in Ugandan children: a randomized trial. JAMA. 2007:297:2210-9.

26. WHO. Guidelines for the treatment of malaria. 3rd ed. Geneva: World Heath Organization; 2015

27. Okell LC, Cairns M, Griffin JT, Ferguson NM, Tarning J, Jagoe G, et al. Contrasting benefits of different artemisinin combination therapies as firstline malaria treatments using model-based cost-effectiveness analysis. Nat Commun. 2014:5:5606.

28. Bretscher MT, Griffin JT, Hugo P, Baker M, Ghani A, Okell L. A comparison of the duration of post-treatment protection of artemether-lumefantrine, dihydroartemisinin-piperaquine and artesunate-amodiaquine for the treatment of uncomplicated malaria. Malar J. 2014;13(Suppl 1):P19.

29. Yeka A, Lameyre V, Afizi K, Fredrick M, Lukwago R, Kamya MR, et al. Efficacy and safety of fixed-dose artesunate-amodiaquine vs. artemetherlumefantrine for repeated treatment of uncomplicated malaria in Ugandan children. PLOS ONE. 2014;9:e113311.

\section{Submit your next manuscript to BioMed Central and we will help you at every step:}

- We accept pre-submission inquiries

- Our selector tool helps you to find the most relevant journal

- We provide round the clock customer support

- Convenient online submission

- Thorough peer review

- Inclusion in PubMed and all major indexing services

- Maximum visibility for your research

Submit your manuscript at www.biomedcentral com/submit
Ciomed Central 